\title{
Intact fetal ovarian cord formation promotes mouse oocyte survival and development
}

\author{
Cory R Nicholas, Kelly M Haston, Renee A Reijo Pera
}

\begin{abstract}
Background: Female reproductive potential, or the ability to propagate life, is limited in mammals with the majority of oocytes lost before birth. In mice, surviving perinatal oocytes are enclosed in ovarian follicles for subsequent oocyte development and function in the adult. Before birth, fetal germ cells of both sexes develop in clusters, or germline cysts, in the undifferentiated gonad. Upon sex determination of the fetal gonad, germ cell cysts become organized into testicular or ovarian cord-like structures and begin to interact with gonadal somatic cells. Although germline cysts and testicular cords are required for spermatogenesis, the role of cyst and ovarian cord formation in mammalian oocyte development and female fertility has not been determined.

Results: Here, we examine whether intact fetal ovarian germ and somatic cell cord structures are required for oocyte development using mouse gonad re-aggregation and transplantation to disrupt gonadal organization. We observed that germ cells from disrupted female gonad prior to embryonic day e13.5 completed prophase I of meiosis but did not survive following transplantation. Furthermore, re-aggregated ovaries from e13.5 to e15.5 developed with a reduced number of oocytes. Oocyte loss occurred before follicle formation and was associated with an absence of ovarian cord structure and ovary disorganization. However, disrupted ovaries from e16.5 or later were resistant to the re-aggregation impairment and supported robust oocyte survival and development in follicles.

Conclusions: Thus, we demonstrate a critical window of oocyte development from e13.5 to e16.5 in the intact fetal mouse ovary, corresponding to the establishment of ovarian cord structure, which promotes oocyte interaction with neighboring ovarian somatic granulosa cells before birth and imparts oocytes with competence to survive and develop in follicles. Because germline cyst and ovarian cord structures are conserved in the human fetal ovary, the identification of genetic components and molecular mechanisms of pre-follicle stage germ and somatic cell structures may be important for understanding human female infertility. In addition, this work provides a foundation for development of a robust fetal ovarian niche and transplantation based system to direct stem cellderived oocyte differentiation as a potential therapeutic strategy for the treatment of infertility.
\end{abstract}

\section{Background}

Ovarian follicles, consisting of an oocyte and surrounding somatic granulosa cells, are essential for oocyte survival and maturation [1,2]; however, the role of prefollicle stage fetal ovarian germ and somatic cell structures in directing mammalian oocyte development has not been well defined. Prior to follicle formation, germ cell precursors of several species have been observed to develop in cysts $[3,4]$. Germline cyst formation occurs by incomplete cytokinesis during mitosis, resulting in

\footnotetext{
* Correspondence: reneer@stanford.edu

Institute for Stem Cell Biology and Regenerative Medicine, Department of Obstetrics and Gynecology, Stanford University, Palo Alto, CA, USA
}

the connection of daughter cells by intercellular bridges. In Drosophila, cyst formation and intercellular bridgemediated transport of organelles and RNA determine oocyte fate and fertility of the adult female [5-7].

Mouse primordial germ cells (PGCs) also develop in cyst-like clusters following colonization of the genital ridge and subsequent cell division. The maximum number of germ cell clusters is detected on embryonic day e13.5 just prior to commitment, or maturation, of PGCs to an oocyte developmental program and successive entry into meiosis [8]. Upon commitment, oocyte clusters become organized into poorly defined ovarian, or ovigerous, cord-like structures containing oocytes and

\section{()


pre-granulosa somatic cells [1]. Shortly after birth, ovarian cords and cysts break down into follicles characterized by oocyte apoptosis, the organization of granulosa cells around surviving oocytes, and basement membrane remodeling [9-11]. Follicle formation therefore appears to require intricate synchronization of oocyte precursor germ cells and pre-granulosa somatic cells [12,13].

Although ovarian cyst and cord break down has been implicated in follicle formation [10], it is not yet known whether cyst and/or cord formation is required for mammalian oocyte development before follicle formation. Previously, intercellular bridges were found to be dispensable for female fertility in mammals [14]; however, germ cell cyst formation was not obstructed in mice lacking bridges, and, therefore, the requirement of cyst and cord formation for oocyte development could not be determined. Here, we use fetal ovary re-aggregation and transplantation to directly perturb ovarian cysts and cords, and we demonstrate that intact ovarian cord formation and maturation promote oocyte survival and development.

\section{Results}

\section{Intact ovary maturation promotes oocyte development}

To investigate the role of intact ovarian cords in promoting oocyte development, we dissected fetal to newborn stage mouse female genital ridges and ovaries, dissociated the tissues to single cells, re-aggregated the dissociated cells, and then transplanted the re-aggregated tissues under the kidney capsules of bilaterally ovariectomized immuno-deficient mouse recipients for three weeks. Post-transplantation, we constructed a timeline denoting the competence of gonads at different stages of development to support oocyte survival and maturation. For this purpose, transplanted grafts from e11.5 to post-natal P2 female gonadal stages were sectioned and analyzed for the presence of oocyte-containing follicles by hematoxylin and eosin staining following re-aggregation and transplantation as described [15]. Follicles containing oocytes with visible nuclei were counted on every $10^{\text {th }}$ section, and the average follicle number per graft ( $n=3$ grafts per gonadal stage) was normalized either per section or per weight (mg) of graft tissue. No significant difference was found between normalization methods (per weight normalization not shown).

We observed that the number of oocytes in follicles detected following re-aggregation and transplantation was not equal and generally increased with the age of embryos used as ovary donors (Figures 1A and 2A). Oocytes that were not enclosed in follicles were never detected by VASA immunostaining analysis of any ovarian stage following three weeks of transplantation (data not shown). In contrast to significant folliculogenesis following transplantation of intact gonadal controls (Figure 1B) or endogenous adult ovary in vivo controls (Figure 1C), ovarian follicles were not detected from re-aggregated e11.5 or e12.5 female genital ridges (Figures $1 \mathrm{~A}$ and $2 \mathrm{~A}$ ). Rare oocytes in follicles were observed beginning from reaggregated e13.5 ovaries, and follicle number increased from e13.5 to e16.5, with a significant elevation from e15.5 to e16.5 (Figure 2A). Robust folliculogenesis was supported by re-aggregated e16.5 to P2 ovarian stages.

E11.5 to e14.5 transplants were then sectioned through and examined in their entirety to ensure that the absence of follicles observed before e13.5 could not be due to dilution of oocytes by ovarian somatic cells that had greater proliferative potential at these earlier gonadal stages. We confirmed an absence of follicles from e11.5 and e12.5 transplants and detected similarly low numbers of oocytes from e13.5 and e14.5 samples (data not shown). In fact, the average weight of grafts was nearly identical between e11-12.5 and e17.5-P2 gonadal stage transplants (Figure 2B). Furthermore, the re-aggregation impairment of oocyte development from early ovarian stages was not a non-specific effect of physical gonadal disruption but rather a biological function of gonadal stage prior to the disruption, as oocyte development occurred following re-aggregation and transplantation of later stage ovaries that were treated identically. To rule out the possibility of later stage ovary resistance to enzymatic dissociation to single cells as being responsible for robust oocyte development, newborn ovaries were dissociated as described, then filtered through a $40 \mu \mathrm{m}$ membrane to remove cell clumps, and FACS sorted for single cells based on forward and side scatter parameters to exclude doublets and to ensure a single cell suspension before re-aggregation. Indeed, FACS sorted and re-aggregated newborn ovaries still supported robust oocyte development of equivalent numbers of follicles after transplantation (36 follicles/section for P2 and P2 FACS samples) (Figure 1D). Thus, the results of the re-aggregation time course indicated that pre-e13.5 stage gonadal cells were not yet competent to support oocyte development following disruption. We concluded that intact ovarian development on e13.5 was important for oocyte survival and maturation in follicles. Intact ovaries are not required for oocyte meiotic entry or progression

Re-aggregated female germ cells became competent to develop as oocytes in follicles after e13.5 and corresponded to the time of female sex determination and PGC commitment to an oocyte developmental program on e13.5 in vivo [16]. Soon after their commitment, oocytes begin to enter meiotic prophase I from e13.5 to e16.5 $[17,18]$, representing an early hallmark of fetal oocyte development and also paralleling the increase in oocyte development observed in our re-aggregation timeline. 

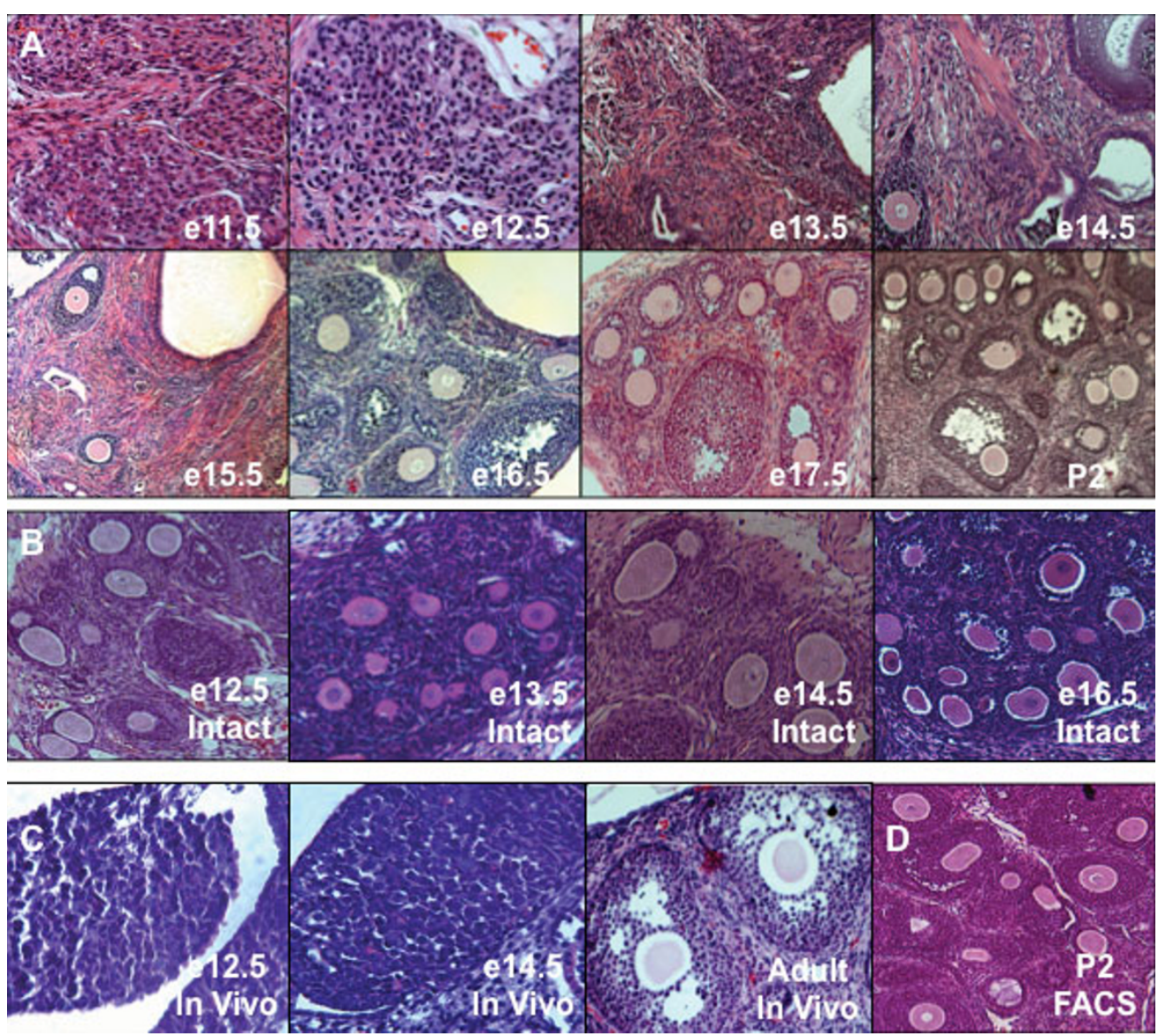

Figure 1 Female gonadal re-aggregation prior to ovary maturation impaired oocyte development. (A) Female mouse genital ridges and ovaries from e11.5 (embryonic day) to P2 (post-natal day) were disrupted by re-aggregation, transplanted under the kidney capsule for three weeks, and sections were examined by H\&E stain for oocyte development in follicles in comparison to intact (B) gonadal transplant controls. (C) For comparison, in vivo control endogenous time points are shown to illustrate e12.5 genital ridge, pre-follicle formation e14.5 differentiating ovary, and post-follicle formation adult ovary. (D) P2 ovaries were also FACS sorted prior to re-aggregation to ensure a single cell suspension and an equivalent dissociation method. 100x magnification.
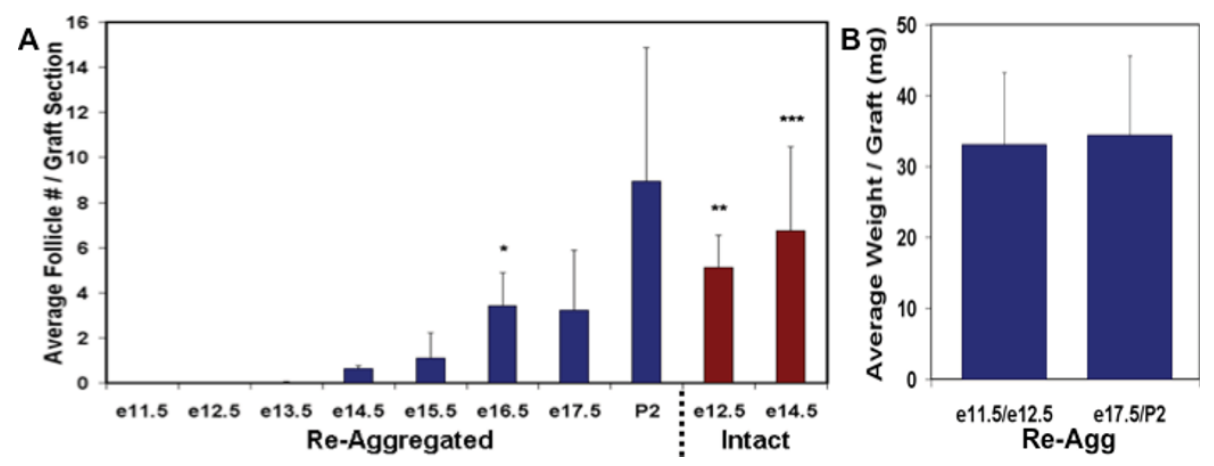

Figure 2 Quantification of re-aggregation induced impairment of oocyte development. (A) The average number of oocytes in follicles per graft, normalized to the number of sections counted, was determined following three weeks transplantation of intact or re-aggregated samples. No oocytes were detected in re-aggregated samples prior to e13.5. Oocyte numbers increased in re-aggregated samples after e13.5 in parallel to the increasing age of ovary donors, however, oocyte numbers were still significantly reduced from e13.5 to e15.5 compared to intact control samples. (B) Average weight per graft following transplantation of re-aggregated early and late gonadal stages demonstrating equivalent weights. Error bars represent s.d. ( $n=3,150$ sections/graft). ${ }^{*}=p<0.05$ between e16.5 and e15.5 re-aggregated. ${ }^{* *} / * *=p<0.05$ between e12.5/e14.5 intact and re-aggregated, respectively. 
Consequently, we examined pre-e13.5 stage germ cells for meiotic entry and progression as a measure of oocyte commitment, and we observed that re-aggregated germ cells were still competent to commit to an oocyte developmental program and enter meiosis following transplantation (Figure 3A). Re-aggregated or intact e12.5 female genital ridges transplanted for three days and assayed by immunofluorescence for expression and localization of synaptonemal complex protein markers of meiotic chromosome synapsis displayed an almost equivalent percentage of meiotic cells. Both samples contained oocytes that expressed markers of zygotene to diplotene stages of meiotic prophase such as chromosomal alignment of SYCP3 (SYnaptonemal Complex Protein $3 ; 59 \%$ in intact and $51 \%$ in re-aggregated), and markers of pachytene to diplotene stages such as chromosomal localization of SYCP1 (45\% in intact and 33\% in re-aggregated) (Figure $3 \mathrm{~B}$ ). Thus, the complete absence of oocytes and follicles from re-aggregated e12.5 samples was not due to lack of oocyte commitment and entry into meiosis.

To determine the extent of meiotic progression, we examined e12.5 genital ridges after five days of transplantation and detected only a small percentage of cells that stained positive for chromosomal elongated localization of SYCP3 and SYCP1 for either re-aggregated or intact control samples (6\% in intact and $14 \%$ in re-aggregated for SYCP3; $4 \%$ in intact and $2 \%$ in re-aggregated for SYCP1) (data not shown). These results indicated that germ cells from both intact and re-aggregated e12.5

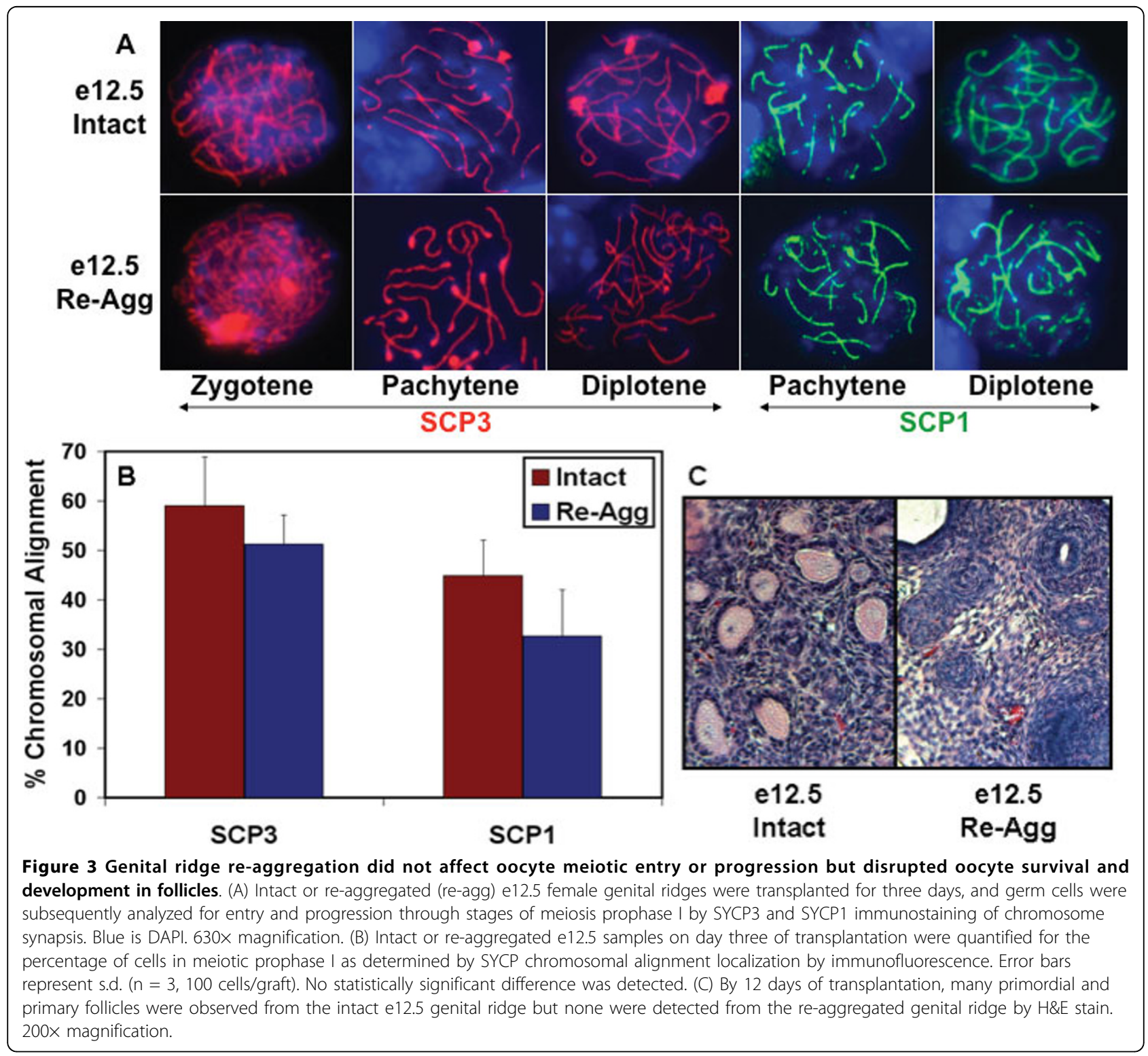


samples entered and progressed through meiotic prophase I to dictyate arrest as oocytes and subsequently down-regulated the expression of SYCP proteins. Downregulation of SYCP1 upon successful meiotic progression of oocytes has been suggested to facilitate follicle formation [19]. Therefore, PGC commitment to oogenesis and oocyte progression through meiotic prophase I did not appear to be affected by re-aggregation, although we cannot eliminate the possibility of defects in DNA mismatch or double strand break repair. In addition, neither complete sex reversal to spermatogenesis and testicular structures nor ectopic PGC reprogramming and teratoma formation following genital ridge disruption were ever observed.

\section{Intact ovary maturation supports oocyte survival before follicle formation}

Although germ cells entered and progressed through early stages of meiosis as committed oocytes in the three to five day transplants from re-aggregated e12.5 female genital ridge, the oocytes did not survive at three weeks of transplantation. We hypothesized that reaggregation either directly impaired oocyte survival or impaired the development of ovarian somatic cells and indirectly led to oocyte degeneration subsequent to an obstruction in the formation of follicles. Thus, intact and re-aggregated e12.5 genital ridges were examined along a time course of transplantation to determine when oocyte loss occurred with respect to follicle formation and ovarian somatic granulosa cell development, and to elucidate a germ cell or somatic cell basis for the re-aggregation impairment of oocyte survival.

Endogenous ovarian follicle formation occurs just after birth in mice. As expected, follicles were not detected from either intact or re-aggregated e12.5 samples after only five to seven days of transplantation, comparable to e17.5 to e19.5 pre-natal stages; however, many primordial follicles were identified from the intact e12.5 female genital ridge beginning on day 12 of transplantation. In contrast, ovarian follicles were not observed from the re-aggregated e12.5 genital ridge on day 12 (Figure 3C). To further characterize oocyte loss, we investigated the decline in oocyte number by TRA98 oocyte marker immunostaining of intact and re-aggregated e 12.5 female genital ridges after transplantation for five, seven, and 12 days. Intact control transplants exhibited gradual oocyte loss during the time course, similar to oocyte loss in vivo, with $20 \%$ of oocytes from day five of transplantation surviving to day 12 and forming follicles. Previously, endogenous peri-natal oocyte loss in vivo was reported to occur through programmed ovarian germ cell cyst break down, with $33 \%$ of oocytes surviving to form primordial follicles $[10,20]$. In contrast, re-aggregation resulted in accelerated oocyte loss and significant reduction in oocyte number by day seven of transplantation with no oocytes surviving to day 12 (Figure 4A). Thus, oocytes from re-aggregated genital ridge were being lost by day seven of transplantation before follicle formation on day 12 and suggested an oocyte-specific impairment as a cause rather than a consequence of folliculogenesis obstruction. Furthermore, FOXL2+ ovarian somatic granulosa cells survived and were detected at all time points of re-aggregated transplantation in similar numbers to intact samples despite the absence of TRA98+ oocytes, in line with prior observations [21,22], confirming a re-aggregation induced oocyte-specific deficiency (Figure 4C).

To determine the mechanism of oocyte loss after reaggregation, we examined markers of apoptosis following transplantation of intact or re-aggregated e12.5 female genital ridge and detected evidence of Caspaseindependent apoptosis. Although activated Caspase2 and 3 apoptotic pathways have been previously implicated in oocyte death [23-29], TRA98+ oocytes expressing active Caspase 2 or Caspase 3 were not detected following reaggregation. However, $2 \%$ of oocytes expressed active Caspase 2 in intact genital ridge, and active Caspase 3 was observed in some FOXL2+ granulosa cells from both samples (Figure 4B, C). In contrast, we detected no TRA98/TUNEL double positive apoptotic oocytes in the intact samples after five days of transplantation, while $1.7 \%$ of the TRA98+ oocytes were TUNEL+ in the reaggregated genital ridge (Figure 4B, B'). On day seven, we detected $2 \%$ and $2.7 \%$ TUNEL+ apoptotic oocytes in intact and re-aggregated samples, respectively (Figure 4B, B"). Therefore, a small increase in TUNEL+ apoptotic oocytes was observed following re-aggregation indicating that TUNEL+, Caspase-independent apoptosis may play a role in re-aggregation induced oocyte loss. However, other mechanisms of cell death or loss, such as necrosis and/or autophagy, are likely to be involved in oocyte reduction/depletion as well. Additionally, oocyte differentiation can not be excluded.

\section{Intact ovarian cord formation promotes oocyte survival and development}

Having demonstrated that oocytes are directly impaired and lost before follicle formation following disruption of the e12.5 female genital ridge, we next explored the properties of the ovary that develop on e13.5 and enable oocyte survival after re-aggregation and transplantation. We observed that re-aggregation resulted in the disorganization of ovary structure and corresponded to the absence of ovarian cord formation and subsequent oocyte loss. In transplants of intact e12.5 female genital ridge, TRA98+ oocytes and FOXL2+ granulosa cells colocalized to large clusters or ovarian cords by five days of transplantation that correlated with successive oocyte survival and follicle formation on day 12 . However, ovarian cords were significantly disrupted in transplants 


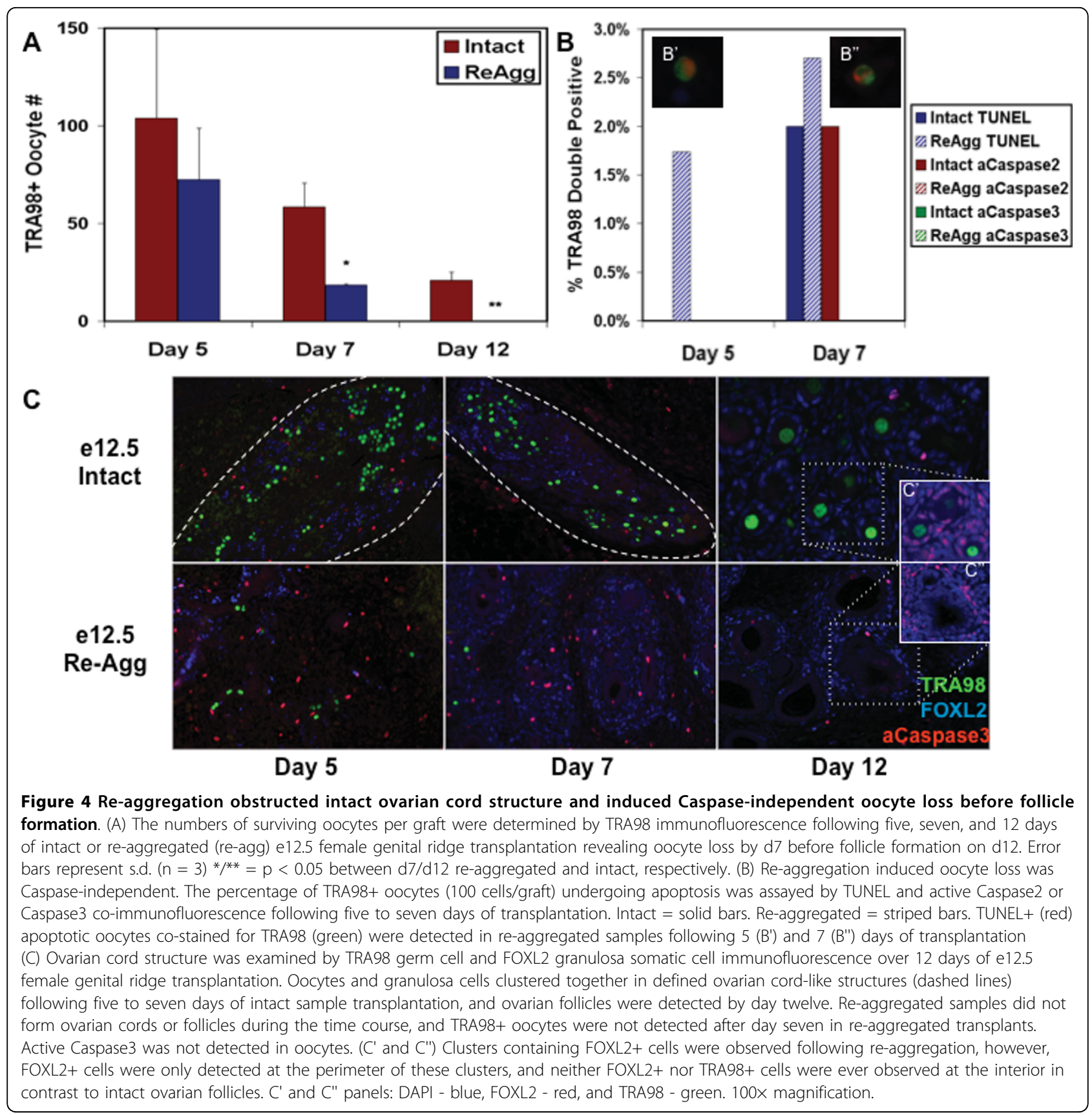

from re-aggregated e12.5 female genital ridge, as clusters of TRA98+ oocytes and FOXL2+ granulosa cells were not observed at any time point after transplantation (Figure 4C). Instead, individual TRA98+ oocytes were distributed evenly throughout the re-aggregated gonad after five to seven days of transplantation and were not detected by day 12 (Figures $4 \mathrm{C}$ and 5).

In contrast, FOXL2+ granulosa cells were found to reorganize in clusters following re-aggregation, however, these granulosa cell domains were not specifically enriched for oocytes and exhibited a distinctly different morphology from intact ovarian follicles. In contrast to FOXL2+ granulosa cells throughout the somatic cell layers of intact ovarian follicles and adjacent to the oocyte, FOXL2+ cells were confined to the perimeter of these re-aggregated clusters with several layers of FOXL2-, DAPI+ cells at the interior facing an oocyteless lumen (Figure 4C', C'). Oocytes were occasionally observed in a FOXL2+ cluster, but this non-specific interaction was the result of random oocyte distribution and dense granulosa cell domains after disruption and re-aggregation (Figure 5). Thus, the survival and 


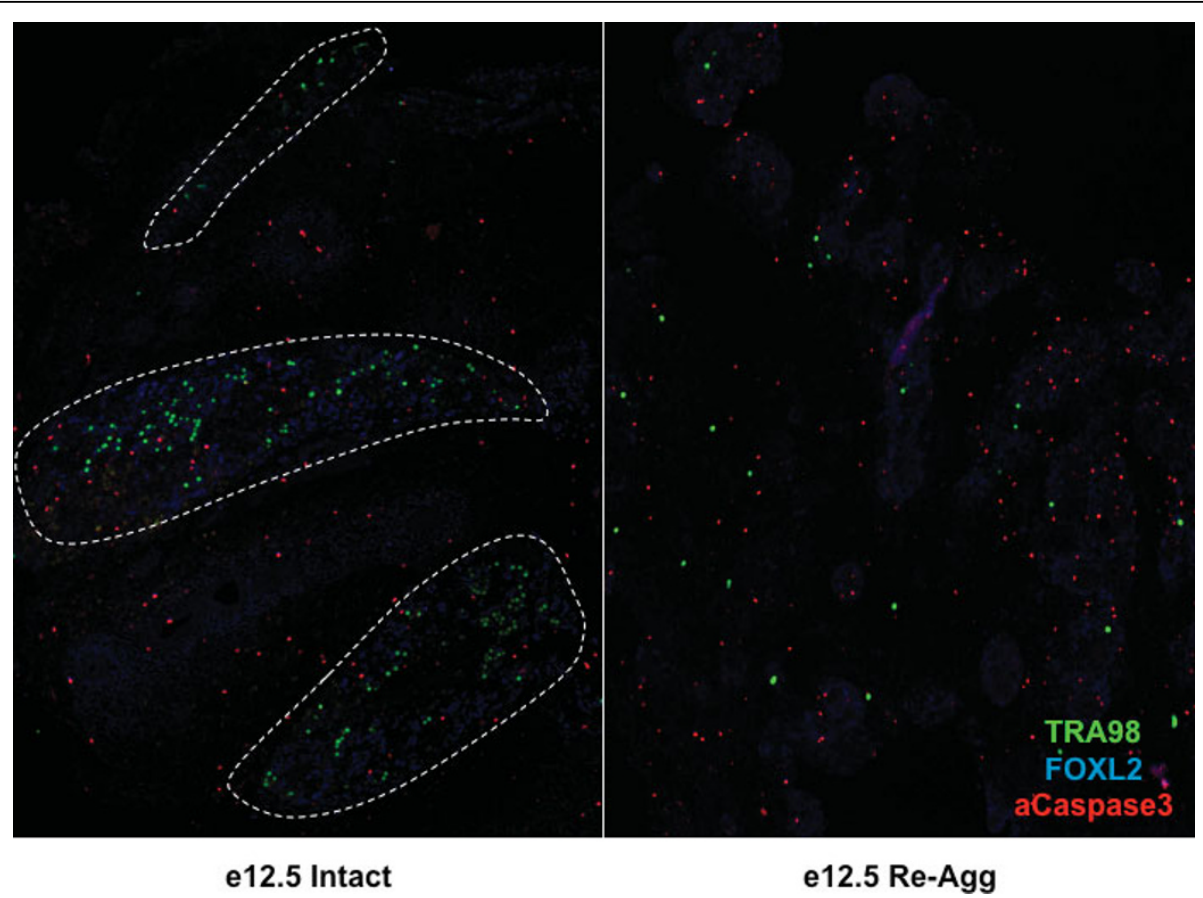

Figure 5 Re-aggregation impaired intact ovarian cord formation. Low magnification (40x) analysis of ovary structure by TRA98 germ cell and FOXL2 granulosa somatic cell immunofluorescence after five days of e12.5 female genital ridge transplantation revealed the striking difference in ovary organization between intact and re-aggregated samples. Intact sample oocytes and granulosa cells clustered together in defined ovarian cord-like structures (dashed lines), in contrast to re-aggregated samples which did not form ovarian cords containing oocyte clusters. Active Caspase3 was not detected in oocytes.

re-organization of granulosa cells, and oocyte lack thereof, was indicative of an oocyte-specific deficiency induced by re-aggregation that corresponded to disruption of ovarian cord formation.

Re-aggregation also resulted in ovary disorganization of other somatic cell types that correlated with ovarian cord disruption and oocyte loss. In intact e12.5 female genital ridge transplants, somatic cells expressing PECAM (Figure 6A) and Laminin (Figure 6B), markers of endothelial cells and basement membrane, respectively, were predominantly separated from oocytes by intact ovarian cords. Of note, we did not detect PECAM expression in oocytes following five days of e12.5 genital ridge transplantation (equivalent to e17.5), confirming a prior report that PECAM expression is extinguished in oocytes by e16 and, thus, can be used as a somatic cellspecific marker at this stage [30]. In re-aggregated samples, in contrast, somatic cells expressing PECAM and Laminin were instead uniformly interspersed, along with randomly dispersed oocytes and granulosa cell clusters, by day five of transplantation (Figure 6A, B). Therefore, in addition to disruption of ovarian cords, disorganized somatic cell contact- and/or paracrine signalingmediated oocyte toxicity induced by the re-aggregation could also play a role in the lack of oocyte survival.
To distinguish ovarian cord disruption from somatic cell toxicity mechanisms of re-aggregation-induced oocyte loss, e12.5 female genital ridges were again dissociated to obstruct ovarian cord formation. Instead of reaggregation, germ cells were purified from e12.5 somatic cells, which may be inhibitory, and were then co-aggregated with later stage e16.5 and P2 ovaries. Notably, we previously found disrupted and re-aggregated e16.5 and P2 ovarian stages capable of supporting robust oocyte survival and development as characterized by ovarian somatic cell organization and folliculogenesis (Figures $1 \mathrm{~A}$ and 2A). Therefore, the inability of e12.5 germ cells to survive as oocytes and contribute to follicle formation and development with later stage ovarian somatic cells would reflect an oocyte-specific deficiency. As such, we used a transgenic Oct4-GFP mouse model [31] to purify e12.5 germ cells from the female genital ridge by fluorescence activated cell sorting (FACS). Purified GFP+ e12.5 germ cells were then directly co-aggregated with e16.5 and P2 ovarian tissues and transplanted for three weeks. Following transplantation, however, we could not identify any GFP+ germ cell-derived oocytes (data not shown) indicating a lack of germ cell-specific competency at the e12.5 stage to support oocyte survival and development. 


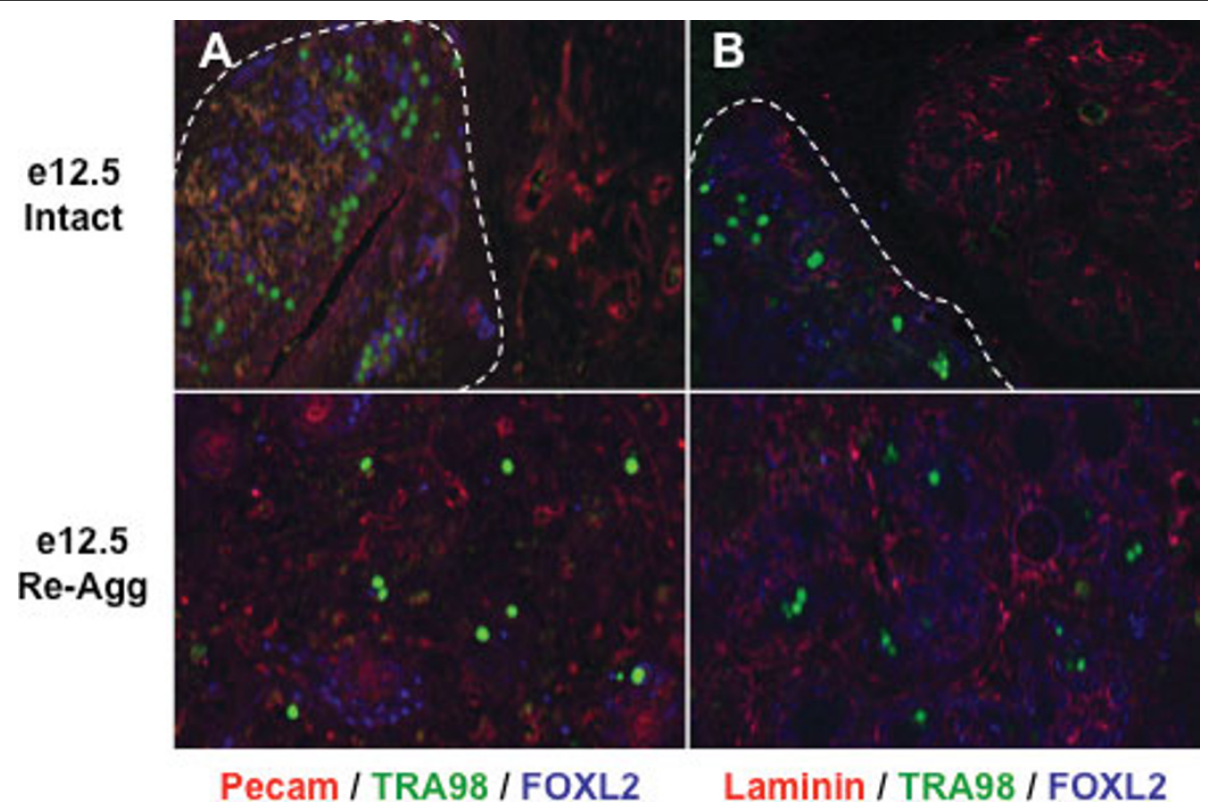

Figure 6 Oocyte loss was associated with ovary disorganization following re-aggregation. (A, B) Immunofluorescence revealed TRA98+ oocytes and FOXL2+ granulosa cells in ovarian cord-like structures (dashed lines) primarily sequestered from PECAM+ endothelial cells (A) and Laminin+ basment membrane (B) following five days of intact e12.5 female genital ridge transplantation. Along with disrupted ovarian cord formation, re-aggregation also resulted in a uniform distribution of PECAM+ and Laminin + cells amongst dispersed oocytes and granulosa cells that correlated with subsequent oocyte loss. 100x magnification.

Although our results advocated an oocyte-specific impairment, we next sought to rule out e12.5-derived oocyte loss due to unsynchronized germ and somatic cell stages. Previous studies suggested that pre-natal oocyte development requires synchronization of germ and somatic cells $[12,13]$, in contrast to post-natal stages where synchronization is not required [32]. Accordingly, e12.5 Oct4-GFP+ germ cells were isolated by FACS and synchronized with later stage ovarian somatic cells by in vitro culture for three days to induce oocyte entry into and progression through meiosis that corresponded to an e15-17.5 stage of ovary development. GFP+ germ cells were cultured on cell culture inserts in media supplemented with several factors adapted from a prior report of in vitro PGC survival and entry into meiosis in the absence of somatic cells [33]. Purified germ cells were alkaline phosphatase positive and continued to express GFP in vitro, confirming their germ cell identity (Figure 7). Furthermore, $78 \%$ of germ cells had entered meiosis by day three of culture as determined by SYCP3 and SYCP1 expression and elongated chromosomal localization, indicating a zygotene to pachytene stage of meiosis prophase I and confirming oocyte maturation in vitro. Meiotic oocytes were subsequently co-aggregated with permissive e16.5 or P2 ovaries and transplanted. However, we were still unable to detect e12.5 germ cell-derived GFP+ oocyte survival and development even though many GFP- oocytes from e16.5 and
P2 ovaries were observed in follicles (Figure 7). Thus, we confirmed a re-aggregation-induced, e12.5-derived deficiency that was oocyte-intrinsic and corresponded to a disruption of intact ovarian cord formation, demonstrating a critical window from e13.5 to e16.5 of oocyte development in the intact ovary for subsequent oocyte survival and maturation in follicles.

Furthermore, preliminary results have been obtained to further confirm an oocyte-specific impairment by performing a reverse experiment. Ovarian tissue from permissive e16.5 embryos was co-aggregated with somatic mesonephros tissue from non-permissive e13.5 ovaries and transplanted for 12 days. Oocytes from e16.5 ovaries have already been exposed to intact ovarian cord structures beginning at e13.5 of development. Based on our previous results suggesting oocyte survival following re-aggregation to be an oocyte-intrinsic function of intact ovarian cord development from e13.5 to e16.5, we expected that these e16.5 oocytes would be competent to survive and form follicles, even when in the presence of somatic tissue from the genital ridges of pre-competent stages. Indeed, e16.5 oocytes survived to form follicles and further suggested an oocyte-intrinsic impairment following the disruption of intact ovarian cord structure during early genital ridge and ovarian stages (data not shown). Nevertheless, we do not exclude the possibility that re-aggregated somatic cells from the early female genital ridge and ovary contribute 


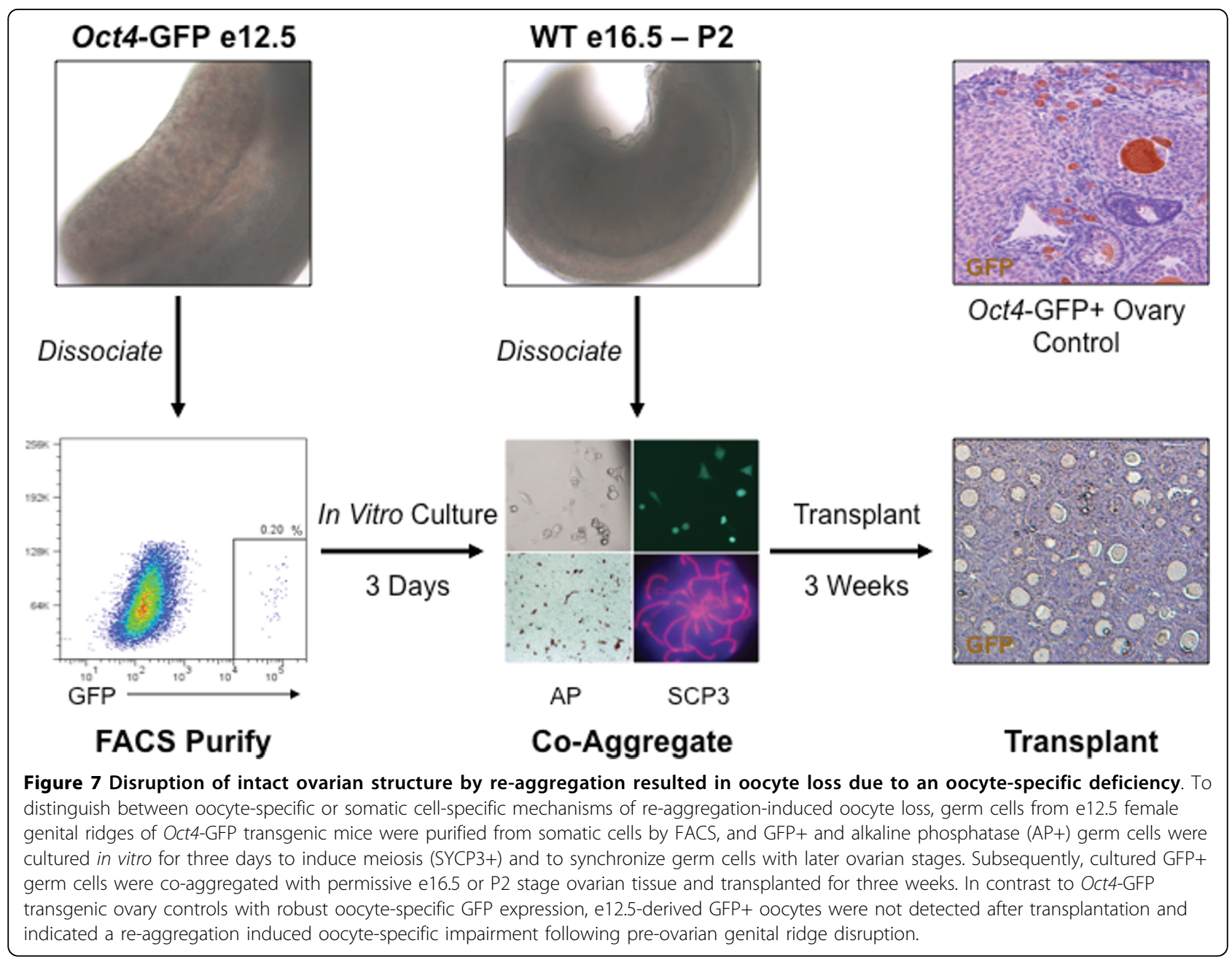

to the inhibition of oocyte survival and development along with the absence of intact ovarian cords.

\section{Discussion}

In the present study, we demonstrate that intact ovarian cord formation promotes oocyte development. The female embryonic genital ridge was not competent to support oocyte survival when disrupted prior to e13.5 ovary maturation and cord formation. Furthermore, oocyte competence to survive and develop in follicles following ovary re-aggregation paralleled the duration of oocyte development within intact fetal ovarian cords from e13.5 to e16.5 prior to their disruption. Pre-ovarian genital ridge re-aggregation did not affect germ cell sex determination or commitment to oogenesis and entry into meiosis (Table 1). However, pre-ovarian e12.5 re-aggregation triggered oocyte loss before follicle formation, which was independent of Caspase pathway activation, and was correlated with obstruction of ovarian cord formation and ovary disorganization. In contrast, granulosa cells survived re-aggregation and exhibited re-organization, indicating an oocyte-specific deficiency. Moreover, heterochronic experiments of preovarian stage germ cell co-aggregation with later stage ovary somatic cells, that were competent to direct follicle formation following re-aggregation, did not support e12.5-derived oocyte survival and confirmed a critical role for e13.5-e16.5 intact ovarian structures in programming oocytes with intrinsic competence to survive and develop in follicles.

Based on our results, we suggest a model in which intact ovarian cord structures that develop on e13.5 provide factors that support oocyte development (Figure 8). A notable aspect of intact ovary maturation is the development of oocytes in clusters which may facilitate cell contact and/or paracrine signal support of oocyte survival. In a previous study, intercellular bridges were not required for oocyte cyst formation and female fertility, although their absence resulted in significant peri-natal oocyte loss [14]. However, germ cell contacts have also been reported to occur by cadherin protein adhesion in vivo and in vitro as early as the PGC migratory stage 
Table 1 Summary of analysis and results following e12.5 female genital ridge transplantation.

\begin{tabular}{|c|c|c|c|c|}
\hline $\begin{array}{l}\text { Days of } \\
\text { Transplantation } \\
\end{array}$ & Analysis $^{a}$ & Re-Agg Gonad & Intact Gonad & In Vivo ${ }^{\text {b }}$ Control \\
\hline 3 & Meiosis & $\begin{array}{c}\text { Zygotene-pachytene of } \\
\text { meiosis I }\end{array}$ & Zygotene-pachytene of meiosis I & Zygotene-pachytene of meiosis I [18] \\
\hline 5 & $\begin{array}{l}\text { Ovarian Cord } \\
\text { Formation }\end{array}$ & $\begin{array}{l}\text { Ovarian cords do not form } \\
\text { and ovary disorganized }\end{array}$ & $\begin{array}{l}\text { Ovarian cords form with distinct } \\
\text { organization of oocyte and } \\
\text { granulosa cell clusters }\end{array}$ & $\begin{array}{l}\text { Ovarian cords form with distinct } \\
\text { organization of oocyte and granulosa } \\
\text { cell clusters [1] }\end{array}$ \\
\hline 7 & Oocyte Survival & $\begin{array}{l}\text { Oocyte numbers significantly } \\
\text { reduced compared to } \\
\text { intact gonad }\end{array}$ & $\begin{array}{l}\text { Oocyte numbers reduced compared } \\
\text { to day } 5\end{array}$ & $\begin{array}{l}\text { Oocyte numbers decline due to } \\
\text { programmed breakdown [10] }\end{array}$ \\
\hline $12-21$ & $\begin{array}{l}\text { Oocyte Development } \\
\text { in Follicles }\end{array}$ & $\begin{array}{c}\text { Oocytes do not survive to } \\
\text { day } 12 \text { and follicles not } \\
\text { observed }\end{array}$ & $\begin{array}{c}\text { Oocytes survive, form follicles, and } \\
\text { mature }\end{array}$ & $\begin{array}{c}\text { Oocytes survive, form follicles, and } \\
\text { mature [2] }\end{array}$ \\
\hline
\end{tabular}

${ }^{a}$ For all experiments, $\mathrm{n}=3$ transplants with 4 embryos or 8 gonads per transplant

${ }^{\mathrm{b}}$ In vivo control refers to e15.5, e17.5, e19.5, and P4 - P14 stages

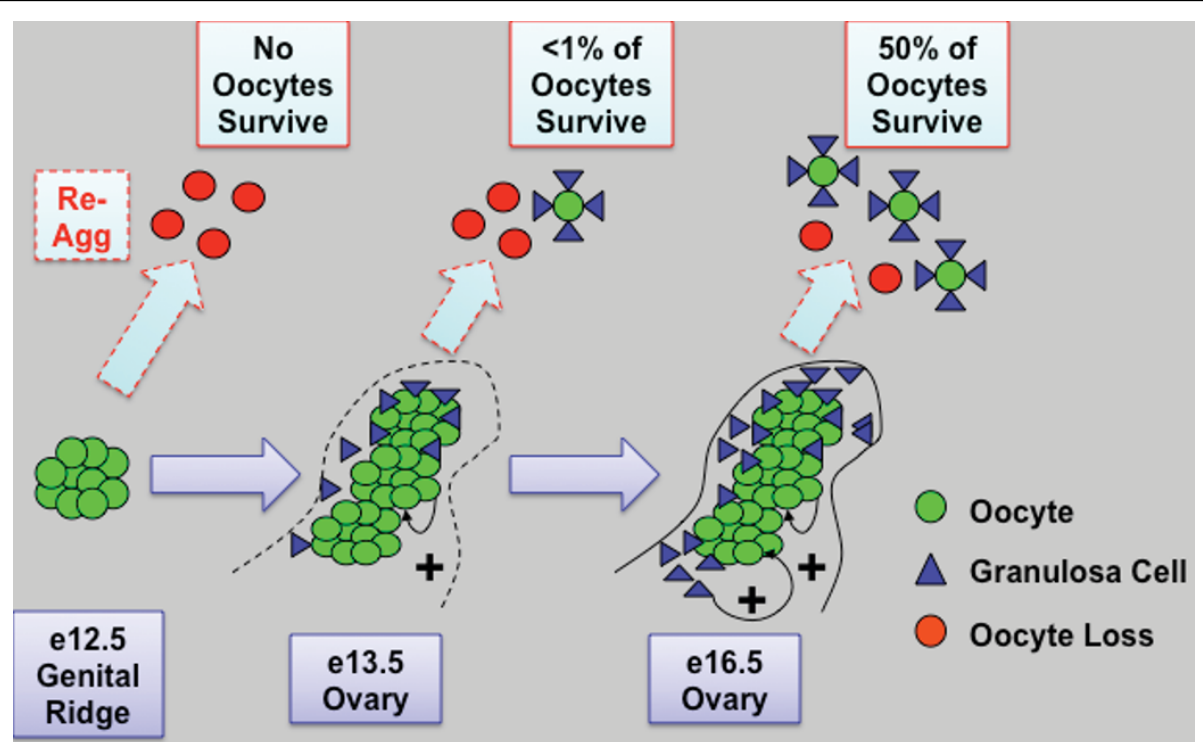

Figure 8 Model of intact ovarian cord formation and maturation promoting oocyte survival and development in follicles. Prior to ovary development on e13.5, germ cells located in cysts in the e12.5 female genital ridge are not yet competent to survive and form follicles when reaggregated (re-agg). However, ovary maturation on e13.5 and development of intact ovarian cord structures containing oocyte and somatic granulosa cell clusters are sufficient to permit some oocyte survival and follicle formation upon re-aggregation. By e16.5, intact ovarian cordenclosed meiotic oocyte clusters and granulosa somatic cells are now primed to undergo robust follicle formation and oocyte development in follicles when re-aggregated. The percentage of surviving re-aggregated oocytes was calculated in comparison to intact transplant controls. Oocyte (green) and granulosa cell (blue) contacts and paracrine signaling factors may promote oocyte survival (+) and possibly facilitate programmed ovarian germ cell cyst break down into follicles. The cord and/or oocyte-mediated recruitment of granulosa cell clusters during ovary differentiation may also provide somatic cell signaling factors such as WNT4, R-spondin1, and Follistatin that promote oocyte survival and maturation. In addition, intact ovarian cords may facilitate the protection of oocytes from re-aggregation induced inhibitory signaling from somatic cells in the genital ridge. Thus, we define a critical window from e13.5 to e16.5 of oocyte enclosure in intact fetal ovarian cord structures for the intrinsic programming of oocytes with competence to survive and undergo further development.

and may account for oocyte cyst-like clustering and survival in the absence of intercellular bridge support [34-36]. Although re-aggregation-mediated disruption of intercellular bridges may have contributed to the reduction in oocyte numbers following transplantation, we attribute the complete absence of oocyte survival to the additional perturbation of ovarian cyst and cord structures. Furthermore, ovarian cords are comprised of pre-granulosa somatic cells, and pre-granulosa-oocyte interactions through cell contacts and/or paracrine factors may promote oocyte survival as well [37]. Cord organization of granulosa cells in close physical proximity to oocytes may also facilitate cyst break down and follicle formation $[10,11,22]$, and the results presented here support this notion. Our results are also in agreement with a previous report of in vitro follicle formation 
from e13.5 ovaries but not from e12.5 genital ridge, confirming an important role for ovarian cord structures that develop in the intact ovary [12].

While disruption of the genital ridge resulted in an oocyte-intrinsic impairment that was independent of somatic granulosa cells, the possibility exists that other types of somatic cells in the re-aggregated genital ridge contributed to oocyte loss. Somatic endothelial and basement membrane cell types expressing PECAM and laminin were inappropriately localized in close proximity to oocytes following re-aggregation. Therefore, in addition to directly imparting oocytes with competence to survive and form follicles, intact ovarian cords that develop on e13.5 may be required to help protect oocytes from deleterious factors expressed by somatic cells in the gonad and/or mesonephros, ensuring oocyte survival and development (Figure 8). Although the mesonephros has traditionally been referenced as a facilitator of female ovary maturation and oocyte meiosis [38-40], the mesonephros also contributes to testicular cord formation and development $[41,42]$. In addition to the presence of meiotic oocytes [43], WNT4, R-spondin1, and Follistatin somatic cell signaling factors were required for ovary sex determination and repressed testicular endothelial and somatic cell migration from the mesonephros [44-48]. Female mice containing genetic null mutations in these factors developed disorganized ovaries with oocytes that entered meiosis but did not survive; analogous to the phenotype reported in this study. The unexplained oocyte loss in these mutant mice therefore may be an indirect consequence of ovary disorganization downstream of the mutation. In the current study, although ovarian somatic cell signaling pathways were not mutated, genital ridge disruption was sufficient to prevent re-organization of ovary structure and potential ovarian cord protection of oocytes from restrictive factors. Future studies will determine genital ridge somatic cell contribution to oocyte loss following re-aggregation and disruption of the ovarian cords.

\section{Conclusions}

In conclusion, we report that intact ovarian cord structures that develop on e13.5 are critical for oocyte development. Thus, we emphasize the significance of intact ovary organization during mid (e13.5) to late (e16.5) gestation for programming oocytes with intrinsic competence to survive and later develop in mouse ovarian follicles after birth. Human oocytes also develop in cystand cord-like structures [49], and identification of genetic mutations and/or environmental toxins affecting human fetal ovary organization could provide insight into lack of human oocyte survival and development in premature ovarian failure-based cases of infertility. Additionally, the understanding of intact fetal ovarian structure and function will facilitate the development of a robust transplantation-based ovarian niche to direct the maturation of oocytes from other potential sources, such as embryonic stem cells, for the treatment of female infertility.

\section{Methods}

Gonad re-aggregation and kidney capsule transplantation The transplantation system was adapted from a previous report of successful oocyte maturation following newborn ovary re-aggregation and transplantation under the kidney capsule [50]. Fetal to newborn stage female gonads with the mesonephros were dissected from wild type CD-1 mice (Charles River) and dissociated to single cell suspensions by a 10 minute $0.25 \%$ trypsinization followed by pipetting 10-20 times and re-suspension in standard media (DMEM, 10\% FBS, 1\% L-glutamine; Invitrogen). For e11.5 to e13.5 stages, genomic DNA was isolated from somatic tissue of each embryo and genotyped for Sry to confirm the gender (Zymo) [51]. The gonadal cell suspension from 4 embryos or pups was then mixed with $0.2 \mathrm{mg} / \mathrm{mL}$ of phytohemagglutinin (Sigma). Cell suspensions were pelleted at 10,000 $\times \mathrm{g}$ for 1 minute, and pellets were incubated overnight in standard media on CM cell culture inserts (Millipore) at $37^{\circ} \mathrm{C}$. Pellets, or grafts, were transplanted under the kidney capsule of bi-laterally ovariectomized CB.17 SCID recipient mice (Charles River) according to the protocol (\#16146) approved by the Stanford University Administrative Panel on Laboratory Animal Care and as described in detail on the NIH Biology of Mammary Gland website link: http://mammary.nih.gov/tools/mousework/Cunha001/Pages/Written_Method.html.

\section{Meiotic cell spread and immunofluorescence}

Following transplantation, tissues were dissociated as above and re-suspended in $20 \mu \mathrm{L}$ of hypo-extraction buffer pH-8.2 (30 mM Tris pH 8.2, $50 \mathrm{mM}$ Sucrose, $17 \mathrm{mM}$ Citric Acid, $5 \mathrm{mM}$ EDTA, $0.5 \mathrm{mM}$ DTT, and $1 \%$ protease inhibitor cocktail (all Sigma)) for $30 \mathrm{~min}$ utes at room temperature. $60 \mu \mathrm{L}$ of $100 \mathrm{mM}$ Sucrose was then added, and the cell suspension was spread onto slides pre-coated with $1 \%$ Paraformaldehyde (USB Corporation) and $0.15 \%$ Triton-X100 (Sigma) in PBS $\mathrm{pH}-9.2$, and dried overnight at room temperature. Slides were blocked in $4 \%$ chicken serum (Abcam) and incubated overnight at $4 \hat{a}-\mid C$ with primary antibody in TBST (tris buffered saline (TBS), 1\% BSA, and 0.1\% Tween-20 (all Sigma)) and 1\% serum. Primary antibodies included rabbit anti-SYCP3 (1:1000, Abcam, ab15092) and rabbit anti-SYCP1 (1:500, Abcam, ab15090). Slides were subsequently incubated with Alexa Fluor chicken anti-rabbit (1:1000, Invitrogen) secondary antibodies for 30 minutes, and cover slips were mounted with Prolong Gold Antifade with DAPI (Invitrogen). 


\section{Immunofluorescence and TUNEL}

Grafts were fixed overnight in 4\% Paraformaldehyde (USB Corporation), embedded in paraffin, and sectioned in 5 to $8 \mu \mathrm{m}$ intervals. In brief, sections on slides were de-paraffinized, re-hydrated, antigens unmasked by incubating in Target Retrieval Solution (Dako) at 95â- IC for 30 minutes, permeabilized in $0.1 \%$ Triton-X100 (Sigma) for 5 minutes, blocked with 10\% chicken serum in TBST overnight, and incubated with primary antibody in TBST with $1 \%$ serum for 1 hour at room temperature. Primary antibodies included anti-VASA rabbit polyclonal (1:500, Abcam, ab13840), anti-TRA98 rat monoclonal (1:500, B-Bridge, 73-003) [52,53], antiFOXL2 goat polyclonal (1:100, Abcam, ab5096), active anti-Caspase2 (ab2251) or anti-Caspase3 (ab13847) rabbit polyclonal (both 1:100, Abcam), anti-CD31 PECAM rabbit polyclonal (1:20, Abcam, ab28364), anti-Laminin rabbit polyclonal (1:200, Abcam, ab11575), and anti-GFP rabbit monoclonal E385 (1:1000, Abcam, ab32146). After washing in TBST, slides were incubated with secondary antibody for 30 minutes at room temperature. Secondary antibodies included Alexa Fluor chicken antirabbit, rat, and goat (1:1000, Invitrogen), and cover slips were mounted with Prolong Gold Antifade with DAPI (Invitrogen). The terminal deoxynucleotidyl transferase dUTP nick end labeling (TUNEL) assay was performed prior to co-immunofluorescence according to the manufacturer's instructions (Roche Diagnostics).

\section{Fluorescence activated cell sorting (FACS)}

Oct4-GFP gonads were dissociated to single cells with $0.25 \%$ Trypsin (Invitrogen) for $5-10$ minutes at $37^{\circ} \mathrm{C}$ and re-suspended in standard media. Cells were strained through a $40 \mu \mathrm{m}$ filter (BD Biosciences) and then analyzed on a BD-FACSAria cell sorting system (BD Biosciences). Post sorting, 10 to 20 thousand GFP+ germ cells were re-plated per well on a 24-well PET $0.4 \mu \mathrm{m}$ cell culture insert (BD Falcon) for three days in standard media supplemented with a germ cell factor cocktail adapted from [33]: mouse SCF $100 \mathrm{ng} / \mathrm{mL}$, mouse SDF1 $20 \mathrm{ng} / \mathrm{mL}$, mouse bFGF $20 \mathrm{ng} / \mathrm{mL}$, mouse BMP4 $50 \mathrm{ng} /$ $\mathrm{mL}$ (all R\&D Systems), N-acetylcysteine $1 \mathrm{mg} / \mathrm{mL}$, Forskolin $5 \mu \mathrm{M}$, Retinoic Acid $1 \mu \mathrm{M}$ (all Sigma), and CYP26 inhibitor R115866 $1 \mu \mathrm{M}$ (Johnson\&Johnson). The GFP+ germ cells were then trypsinized and coaggregated with dissociated wild type e16.5 or P2 ovarian tissue and transplanted as described above.

\section{Statistical analysis}

Data are represented as mean $+/$ - standard deviation (s.d.). Statistical significance was determined by unpaired Student's t-Test of unequal variance. Following transplantation, grafts from at least three separate transplants per fetal/newborn stage were sectioned and sampled by counting oocytes with nuclei in follicles on every tenth section for 150 sections by H\&E stain. Follicle number per graft was normalized per section or normalized per milligram (mg) of graft tissue. For meiotic entry and progression, 100 cells from each of three separate transplants per sample were analyzed for meiotic chromosome synapsis. For oocyte survival, the numbers of TRA98+ oocytes were counted in three separate samples per time point. For apoptotic markers, at least 100 TRA98+ oocytes were assessed for co-expression of active Caspase $2 / 3$ or TUNEL.

\section{Acknowledgements}

We thank members of the Reijo Pera laboratory for assistance, Shawn Chavez and Margaret Fuller for critical review of the manuscript, Teri Longacre and Amarjeet Grewall from the Stanford University Department of Pathology for histology, Ronald Abalos for animal husbandry, Roanna Medina for transplantation training, and John Eppig and Karen Wigglesworth for re-aggregation advice. This work was supported in part by the National Institutes of Health (RO1 HD047721; U54HD055764 as part of the Specialized Cooperative Centers Program in Reproduction and Infertility Research), the California TRDRP (14RT0159;15DT-0187), and the Canadian Institutes of Health Research DRA.

\section{Authors' contributions}

CRN performed study design, all experiments including transplantation and analyses, and drafted the manuscript. $\mathrm{KMH}$ carried out genotyping analysis as well as dissection and FACS sorting assistance, and contributed to experimental design. RARP provided financial support, conceived of the study, and participated in its design. All authors read and approved the final manuscript.

Received: 30 June 2009

Accepted: 8 January 2010 Published: 8 January 2010

\section{References}

1. Byskov AG: Differentiation of mammalian embryonic gonad. Physiol Rev 1986, 66:71-117.

2. Matzuk MM, Burns KH, Viveiros MM, Eppig JJ: Intercellular communication in the mammalian ovary: oocytes carry the conversation. Science 2002, 296:2178-80.

3. Gondos B, Zamboni L: Ovarian development: the functional importance of germ cell interconnections. Fertil Steril 1969, 20:176-89.

4. Pepling ME, de Cuevas M, Spradling AC: Germline cysts: a conserved phase of germ cell development?. Trends Cell Biol 1999, 9:257-62.

5. de Cuevas $M$, Lee JK, Spradling AC: alpha-spectrin is required for germline cell division and differentiation in the Drosophila ovary. Development 1996, 122:3959-68.

6. Lin H, Yue L, Spradling AC: The Drosophila fusome, a germline-specific organelle, contains membrane skeletal proteins and functions in cyst formation. Development 1994, 120:947-56.

7. McKearin D, Ohlstein B: A role for the Drosophila bag-of-marbles protein in the differentiation of cystoblasts from germline stem cells. Development 1995, 121:2937-47.

8. Pepling ME, Spradling AC: Female mouse germ cells form synchronously dividing cysts. Development 1998, 125:3323-8.

9. Mazaud S, Guyot R, Guigon CJ, Coudouel N, Le Magueresse-Battistoni B, Magre S: Basal membrane remodeling during follicle histogenesis in the rat ovary: contribution of proteinases of the MMP and PA families. Dev Biol 2005, 277:403-16.

10. Pepling ME, Spradling AC: Mouse ovarian germ cell cysts undergo programmed breakdown to form primordial follicles. Dev Biol 2001, 234:339-51.

11. Sawyer HR, Smith P, Heath DA, Juengel JL, Wakefield SJ, McNatty KP: Formation of ovarian follicles during fetal development in sheep. Biol Reprod 2002, 66:1134-50.

12. Lei L, Zhang H, Jin S, Wang F, Fu M, Wang H, Xia G: Stage-specific germsomatic cell interaction directs the primordial folliculogenesis in mouse fetal ovaries. J Cell Physiol 2006, 208:640-7. 
13. Qing T, Liu H, Wei W, Ye X, Shen W, Zhang D, Song Z, Yang W, Ding M, Deng $\mathrm{H}$ : Mature oocytes derived from purified mouse fetal germ cells. Hum Reprod 2008, 23:54-61.

14. Greenbaum MP, Iwamori N, Agno JE, Matzuk MM: Mouse TEX14 is required for embryonic germ cell intercellular bridges but not female fertility. Biol Reprod 2009, 80:449-57.

15. Tilly JL: Ovarian follicle counts-not as simple as 1, 2, 3. Reprod Biol Endocrinol 2003, 1:11.

16. McLaren A, Southee D: Entry of mouse embryonic germ cells into meiosis. Dev Biol 1997, 187:107-13.

17. Peters $\mathrm{H}$ : Migration of gonocytes into the mammalian gonad and their differentiation. Philos Trans R Soc Lond B Biol Sci 1970, 259:91-101.

18. Borum K: Oogenesis in the mouse. A study of the meiotic prophase. Exp Cell Res 1961, 24:495-507.

19. Paredes A, Garcia-Rudaz C, Kerr B, Tapia V, Dissen GA, Costa ME, Cornea A, Ojeda SR: Loss of synaptonemal complex protein-1, a synaptonemal complex protein, contributes to the initiation of follicular assembly in the developing rat ovary. Endocrinology 2005, 146:5267-77.

20. Tilly JL: Commuting the death sentence: how oocytes strive to survive. Nat Rev Mol Cell Biol 2001, 2:838-48.

21. Merchant-Larios H, Centeno B: Morphogenesis of the ovary from the sterile W/Wv mouse. Prog Clin Biol Res 1981, 59B:383-92.

22. Guigon CJ, Magre S: Contribution of germ cells to the differentiation and maturation of the ovary: insights from models of germ cell depletion. Biol Reprod 2006, 74:450-8.

23. Arnault E, Tosca L, Courtot AM, Doussau M, Pesty A, Finaz C, Allemand I, Lefevre B: Caspase-2(L), caspase-9, and caspase-3 during in vitro maturation and fragmentation of the mouse oocyte. Dev Dyn 2008, 237:3892-903.

24. Bergeron L, Perez Gl, Macdonald G, Shi L, Sun Y, Jurisicova A, Varmuza S, Latham KE, Flaws JA, Salter JC, et al: Defects in regulation of apoptosis in caspase-2-deficient mice. Genes Dev 1998, 12:1304-14.

25. Fenwick MA, Hurst PR: Immunohistochemical localization of active caspase- 3 in the mouse ovary: growth and atresia of small follicles. Reproduction 2002, 124:659-65.

26. Hanoux V, Pairault C, Bakalska M, Habert R, Livera G: Caspase-2 involvement during ionizing radiation-induced oocyte death in the mouse ovary. Cell Death Differ 2007, 14:671-81.

27. Lobascio AM, Klinger FG, Scaldaferri ML, Farini D, De Felici M: Analysis of programmed cell death in mouse fetal oocytes. Reproduction 2007, 134:241-52.

28. Matikainen T, Perez Gl, Zheng TS, Kluzak TR, Rueda BR, Flavell RA, Tilly JL: Caspase-3 gene knockout defines cell lineage specificity for programmed cell death signaling in the ovary. Endocrinology 2001, 142: 2468-80.

29. Ortiz R, Echeverria OM, Salgado R, Escobar ML, Vazquez-Nin GH: Fine structural and cytochemical analysis of the processes of cell death of oocytes in atretic follicles in new born and prepubertal rats. Apoptosis 2006, 11:25-37.

30. Wakayama T, Hamada K, Yamamoto M, Suda T, Iseki S: The expression of platelet endothelial cell adhesion molecule-1 in mouse primordial germ cells during their migration and early gonadal formation. Histochem Cell Biol 2003, 119:355-62.

31. Anderson R, Fassler R, Georges-Labouesse E, Hynes RO, Bader BL, Kreidberg JA, Schaible K, Heasman J, Wylie C: Mouse primordial germ cells lacking beta1 integrins enter the germline but fail to migrate normally to the gonads. Development 1999, 126:1655-64.

32. Eppig JJ, Wigglesworth K, Pendola FL: The mammalian oocyte orchestrates the rate of ovarian follicular development. Proc Natl Acad SCi USA 2002, 99:2890-4.

33. Farini D, Scaldaferri ML, lona S, La Sala G, De Felici M: Growth factors sustain primordial germ cell survival, proliferation and entering into meiosis in the absence of somatic cells. Dev Biol 2005, 285:49-56.

34. Bendel-Stenzel MR, Gomperts M, Anderson R, Heasman J, Wylie C: The role of cadherins during primordial germ cell migration and early gonad formation in the mouse. Mech Dev 2000, 91:143-52.

35. Di Carlo A, De Felici M: A role for E-cadherin in mouse primordial germ cell development. Dev Biol 2000, 226:209-19.

36. Gomperts M, Garcia-Castro M, Wylie C, Heasman J: Interactions between primordial germ cells play a role in their migration in mouse embryos. Development 1994, 120:135-41.

37. Kidder GM, Mhawi AA: Gap junctions and ovarian folliculogenesis. Reproduction 2002, 123:613-20
38. Bowles J, Knight D, Smith C, Wilhelm D, Richman J, Mamiya S, Yashiro K, Chawengsaksophak K, Wilson MJ, Rossant J, et al: Retinoid signaling determines germ cell fate in mice. Science 2006, 312:596-600.

39. Byskov AG: Does the rete ovarii act as a trigger for the onset of meiosis? Nature 1974, 252:396-7.

40. Byskov AG, Grinsted J: Feminizing effect of mesonephros on cultured differentiating mouse gonads and ducts. Science 1981, 212:817-8.

41. Tilmann C, Capel B: Mesonephric cell migration induces testis cord formation and Sertoli cell differentiation in the mammalian gonad. Development 1999, 126:2883-90.

42. Buehr M, Gu S, McLaren A: Mesonephric contribution to testis differentiation in the fetal mouse. Development 1993, 117:273-81.

43. Yao HH, DiNapoli L, Capel B: Meiotic germ cells antagonize mesonephric cell migration and testis cord formation in mouse gonads. Development 2003, 130:5895-902.

44. Chassot AA, Ranc F, Gregoire EP, Roepers-Gajadien HL, Taketo MM, Camerino G, de Rooij DG, Schedl A, Chaboissier MC: Activation of betacatenin signaling by Rspo1 controls differentiation of the mammalian ovary. Hum Mol Genet 2008, 17:1264-77.

45. Jeays-Ward K, Hoyle C, Brennan J, Dandonneau M, Alldus G, Capel B, Swain A: Endothelial and steroidogenic cell migration are regulated by WNT4 in the developing mammalian gonad. Development 2003, 130: 3663-70.

46. Tomizuka K, Horikoshi K, Kitada R, Sugawara Y, Iba Y, Kojima A, Yoshitome A, Yamawaki K, Amagai M, Inoue A, et al: R-spondin1 plays an essential role in ovarian development through positively regulating Wnt-4 signaling. Hum Mol Genet 2008, 17:1278-91.

47. Vainio S, Heikkila M, Kispert A, Chin N, McMahon AP: Female development in mammals is regulated by Wnt-4 signalling. Nature 1999, 397:405-9.

48. Yao HH, Matzuk MM, Jorgez CJ, Menke DB, Page DC, Swain A, Capel B: Follistatin operates downstream of Wnt4 in mammalian ovary organogenesis. Dev Dyn 2004, 230:210-5.

49. Gondos B, Bhiraleus P, Hobel CJ: Ultrastructural observations on germ cells in human fetal ovaries. Am J Obstet Gynecol 1971, 110:644-52.

50. Eppig JJ, Wigglesworth K: Development of mouse and rat oocytes in chimeric reaggregated ovaries after interspecific exchange of somatic and germ cell components. Biol Reprod 2000, 63:1014-23.

51. McClive PJ, Sinclair AH: Rapid DNA extraction and PCR-sexing of mouse embryos. Mol Reprod Dev 2001, 60:225-6.

52. Sato M, Kimura T, Kurokawa K, Fujita Y, Abe K, Masuhara M, Yasunaga T, Ryo A, Yamamoto M, Nakano T: Identification of PGC7, a new gene expressed specifically in preimplantation embryos and germ cells. Mech Dev 2002, 113:91-4.

53. Tanaka H, Pereira LA, Nozaki M, Tsuchida J, Sawada K, Mori H, Nishimune Y: A germ cell-specific nuclear antigen recognized by a monoclonal antibody raised against mouse testicular germ cells. Int J Androl 1997, 20:361-6.

doi:10.1186/1471-213X-10-2

Cite this article as: Nicholas et al:: Intact fetal ovarian cord formation promotes mouse oocyte survival and development. BMC Developmental Biology 2010 10:2.

\section{Submit your next manuscript to BioMed Central and take full advantage of:}

- Convenient online submission

- Thorough peer review

- No space constraints or color figure charges

- Immediate publication on acceptance

- Inclusion in PubMed, CAS, Scopus and Google Scholar

- Research which is freely available for redistribution

Submit your manuscript at www.biomedcentral.com/submit
( Biomed Central 